\title{
Erratum to: Reproductive Strategies in Planarians: Insights Gained from the Bioassay System for Sexual Induction in Asexual Dugesia ryukyuensis Worms
}

Takanobu Maezawa, Kiyono Sekii, Masaki Ishikawa, Hikaru Okamoto, and Kazuya Kobayashi

\section{Erratum to:}

Chapter 9 in: K. Kobayashi et al. (eds.), Reproductive and Developmental Strategies, Diversity and Commonality in Animals, https://doi.org/10.1007/978-4-431-56609-0_9

In the original version of Chapter 9, the electronic supplementary materials were inadvertently omitted. The electronic supplementary materials videos have been included in the chapter. 